\title{
R-PROCESS ABUNDANCE SIGNATURES
}

\author{
JOHN J. COWAN* \\ Department of Physics and Astronomy, \\ University of Oklahoma, \\ Norman, OK 73019, USA \\ E-mail: cowan@nhn.ou.edu \\ CHRISTOPHER SNEDEN \\ Department of Astronomy and McDonald Observatory, \\ University of Texas, \\ Austin, TX 78712, USA \\ E-mail: chris@verdi.as.utexas.edu
}

\begin{abstract}
Abundance observations indicate the presence of rapid-neutron capture (i.e., $r$ process) elements in old Galactic halo and globular cluster stars. These observations demonstrate that the earliest generations of stars in the Galaxy, responsible for neutron-capture synthesis and the progenitors of the halo stars, were rapidly evolving. Abundance comparisons among large numbers of stars provide clues about the nature of neutron-capture element synthesis both during the earliest times and throughout the history of the Galaxy. In particular, these comparisons suggest differences in the way the heavier (including $\mathrm{Ba}$ and above) and lighter neutron capture elements are synthesized in nature. Understanding these differences will help to identify the astrophysical site (or sites) of and conditions in the $r$-process. The abundance comparisons also demonstrate a large star-to-star scatter in the neutron-capture/iron ratios at low metallicities- which disappears with increasing $[\mathrm{Fe} / \mathrm{H}]$ - and suggests an early, chemically unmixed and inhomogeneous Galaxy. The very recent neutron-capture element observations indicate that the early phases of Galactic nucleosynthesis, and the associated chemical evolution, are quite complex, with the yields from different (progenitor) mass-range stars contributing to different chemical mixes. Stellar abundance comparisons suggest a change from the $r$-process to the slow neutron capture (i.e., $s$-) process at higher metallicities (and later times)in the Galaxy. Finally, the detection of thorium and uranium in halo and globular cluster stars offers a promising, independent agedating technique that can put lower limits on the age of the Galaxy and thus the Universe.
\end{abstract}

*Also at: Department of Astronomy and Mcdonald Observatory, University of Texas, Austin, TX 78712 


\section{Introduction}

The heavy solar system abundances (here, $\mathrm{Z}>30$ ) are formed in neutron capture ( $n$-capture) processes, either the slow $(s-)$ or rapid $(r-)$ process. We show in Figure 1 the solar system - also thought of as cosmic - abundances with the neutron-capture elements highlighted. Abundance observations of these elements in halo stars contain vital clues to the nucleosynthesis history and chemical evolution of the Galaxy, and abundances of radioactive elements can also be utilized to obtain age determinations for the oldest stars, which in turn put lower limits on age estimates for the Galaxy and the Universe (e.g., $[1,2])$.

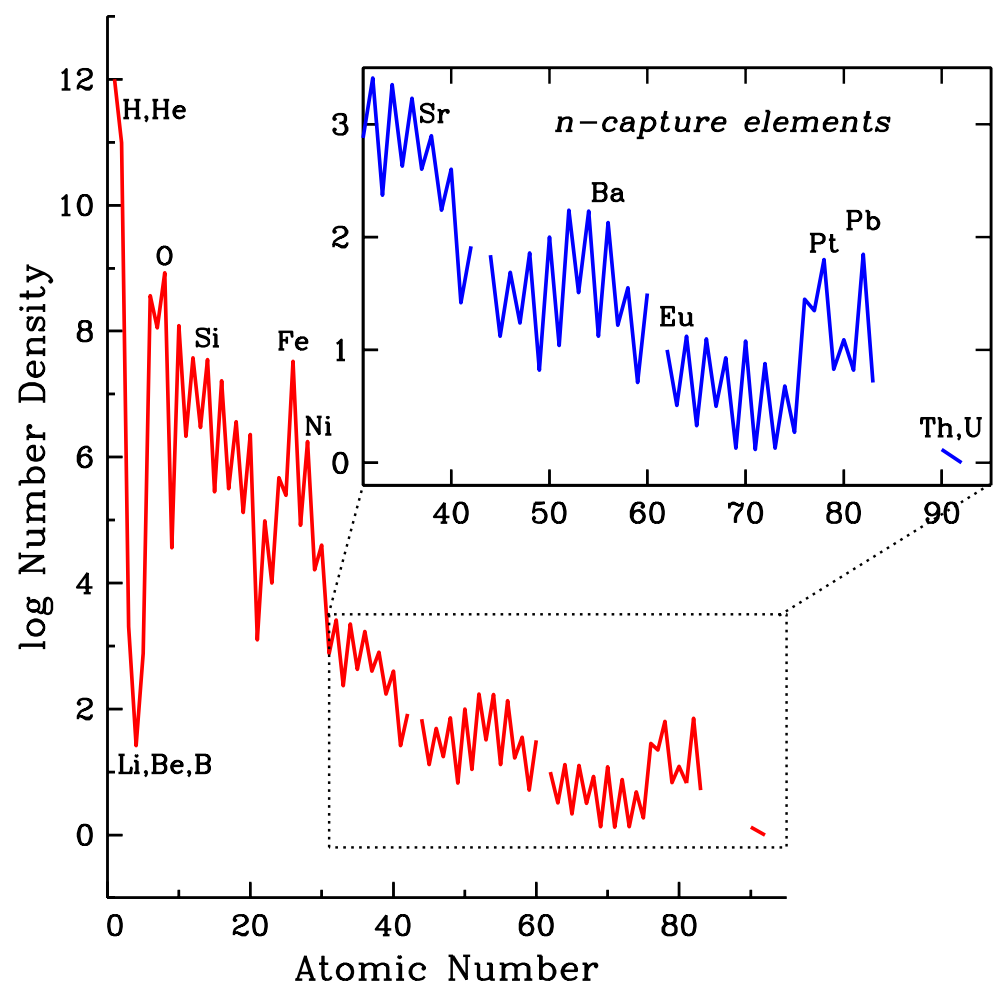

Figure 1. Abundances of elements in the Sun and in undifferentiated solar system material [3]. This abundance set is normalized by convention to $\log \mathrm{N}(\mathrm{H})=12$ in astronomical literature on stars (planetary and meteoritic studies usually normalize the abundances to $\log \mathrm{N}(\mathrm{Si})=6)$. The main figure shows the entire set of stable and long-lived radioactive elements, while the inset is restricted to the $n$-capture elements, defined here as those elements with $\mathrm{Z}>30$. 


\section{Neutron-Capture Abundances in Metal-Poor Halo Stars}

Extensive abundance studies have been made for metal-poor (i.e., low iron abundance) Galactic halo studies [4-7]. We show in Figure 2 detailed abundances for the heaviest $\mathrm{Z}>30 n$-capture abundances in three stars: CS $22892-052, \mathrm{BD}+17^{\circ} 3248$ and HD 115444 [6-8]. The abundances in these stars have been compared to a scaled solar system curve, indicated by the solid line.

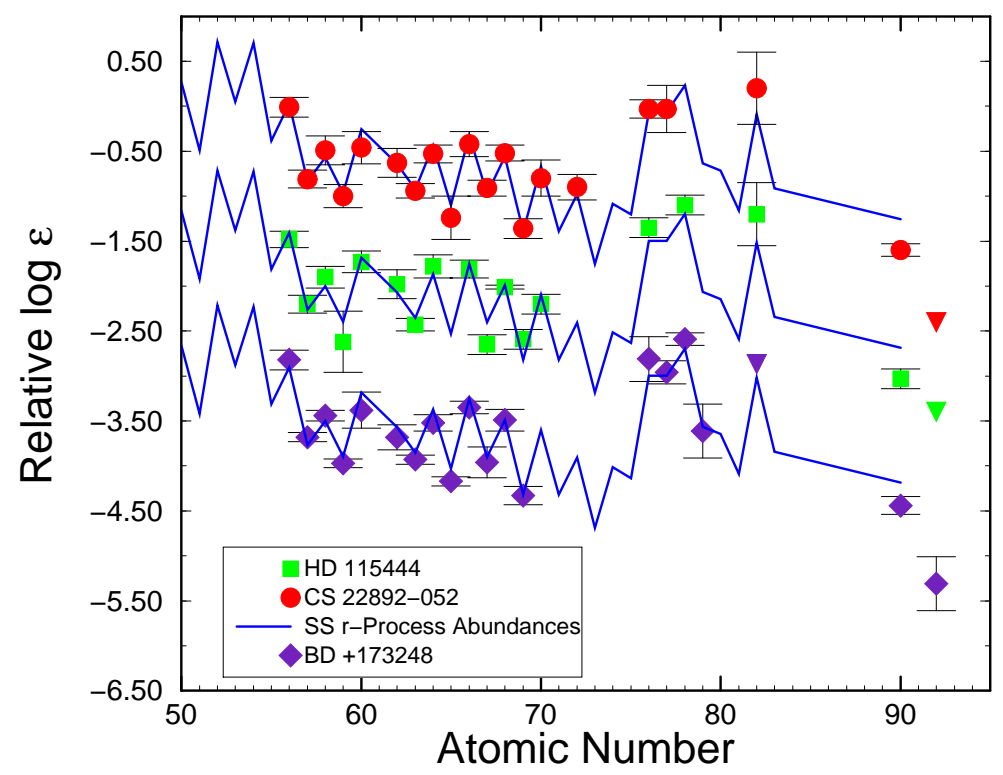

Figure 2. A comparison of observed abundances in the three stars CS 22892-052, HD 115444 and $\mathrm{BD}+17^{\circ} 3248$ with a solar system $r$-process elemental abundances. Upper limits are indicated by inverted triangles.

This solid-line curve was obtained by summing the individual isotopic contributions from the $s$ - and the $r$-process in solar system material from $n$ capture cross section measurements $[9,10]$. These solar elemental $r$-process abundances that were derived are based upon the "classical approach" to the $s$-process, which is empirical and by definition model-independent. Nevertheless, more sophisticated stellar models based upon $s$-process nucleosynthesis in low-mass AGB stars, predict very similar solar abundances [11]. As Figure 2 illustrates there is a remarkable agreement between the solar $r$-process abundances and the abundance patterns of the heaviest $n$ - 
capture elements in these very old metal-poor halo stars. This agreement implies a robust $r$-process, one that seems to reproduce the same relative $n$-capture abundances over many Gyr in the history of the Galaxy. These results further suggest a very narrow range of astrophysical conditions and/or mass ranges for the site(s) for the $r$-process. It has been suggested, for instance, that only low mass $\left(8-10 \mathrm{M}_{\odot}\right)$ supernovae may be a likely site for the main $r$-process and may be responsible for the synthesis of these heavy $(\mathrm{Z} \geq 56) n$-capture elements [12-14].

This agreement between elemental abundances and the solar system $r$ process abundance distribution has now been extended to the isotopic level. Recent results demonstrate that $\mathrm{Eu}$ isotopic abundance fractions in the very metal-poor, $n$-capture-rich giant stars CS 22892-052, HD 115444 and $\mathrm{BD}+17^{\circ} 3248$ are in excellent agreement with each other and with their values in the solar system [15]. The Ba isotopic abundance fractions in one metal-poor star are also consistent with the solar $r$-process values [16], again suggesting the universality of the (main) $r$-process for the heaviest $n$-capture elements.

Until recently there have been little data available on the lighter $n$ capture elements, particularly those from $\mathrm{Z}=40-50$. Recent abundances studies of $\mathrm{BD}+17^{\circ} 3248$ indicated that some of the elements in this regime, specifically the element Ag, seemed to deviate from the same solar curve that fit the heavier $n$-capture elements. This seemed to support an earlier suggestion, based upon solar system meteoritic studies, of two $r$-processes - one for the elements $\mathrm{A} \gtrsim 130-140$ and a second $r$-process for the lighter elements [17]. We show in Figure 3 preliminary new data on CS 22892-052. we note the detection of elements never seen before in this star, including $\mathrm{Mo}, \mathrm{Lu}, \mathrm{Au}, \mathrm{Pt}$ and $\mathrm{Pb}[18]$. Also significant new upper limits have been found for $\mathrm{Ga}, \mathrm{Ge}, \mathrm{Cd}$ and $\mathrm{Sn}$. Comparison of the abundances with the solar $r$-process curve [10] demonstrates the same agreement found previously for the heaviest $n$-capture elements in this star and other similar stars $[6,1,2]$. It is clear from Figure 3 that some of the lighter elements between $\mathrm{Z}=$ 40-50 (e.g., Ag) show significant deviations while others appear to fall near the line. This new result is consistent with earlier studies of this star and with that of $\mathrm{BD}+17^{\circ} 3248$. However, with only a few stars and very limited data available, it is not clear at this point what is the source of the synthesis for these lighter elements. It has been suggested that perhaps, analogously to the $s$-process, the lighter elements might be synthesized in a "weak" $r$ process with the heavier elements synthesized in the more robust "strong" or "main" $r$-process [19]. While a second $r$-process site, perhaps supernovae 
of a a different mass range or frequency [20] or perhaps the helium zone of an exploding supernovae [19], might be responsible for the synthesis of nuclei with $\mathrm{A} \lesssim 130-140$, there have also been suggestions that the entire abundance distribution could be synthesized in a single core-collapse supernova $[6,21]$.

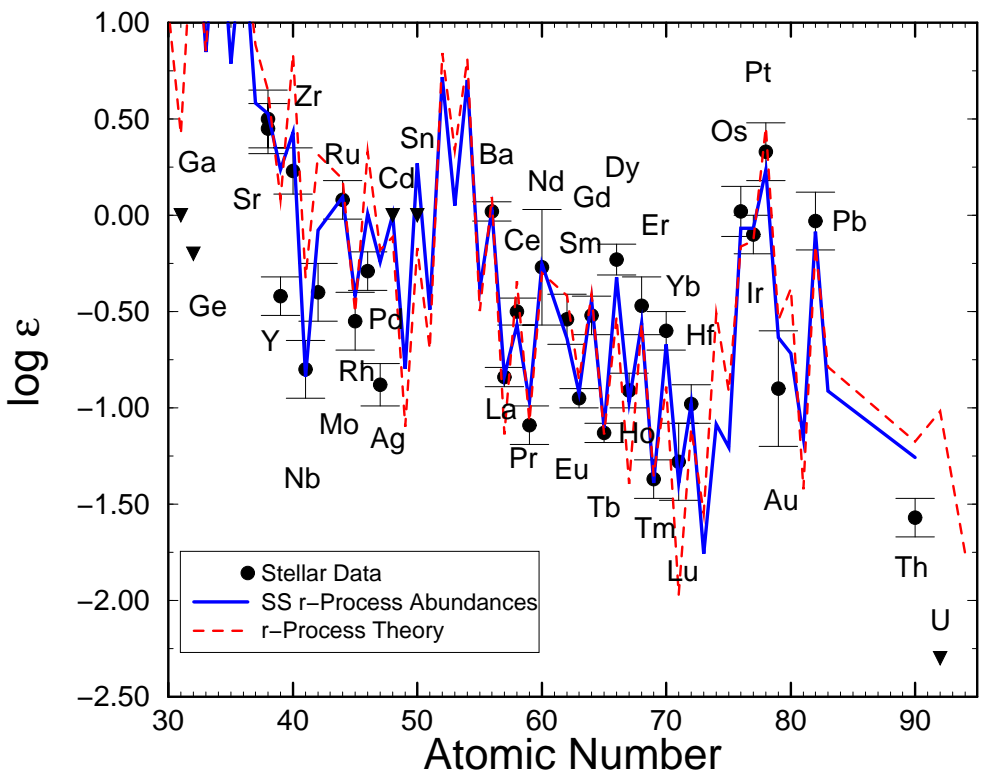

Figure 3. A comparison of new observed abundances from CS 22892-052 with solar system [10] and theoretical [5] $r$-process elemental abundances.

\section{Abundance Scatter and the Chemical Evolution of the Galaxy}

A number of studies have demonstrated a dramatic and very large star-tostar scatter in the abundance level of the heavy $n$-capture elements with respect to the to iron abundances. This star-to-star scatter increases dramatically with decreasing stellar metallicity, as shown in Figure 4, in which we plot $[\mathrm{Eu} / \mathrm{Fe}]^{\mathrm{a}}$ abundance ratios as a function of $[\mathrm{Fe} / \mathrm{H}]$ metallicity for a large number of halo and disk stars $[1,2]$. Eu is employed for such studies

$\overline{\mathrm{a}}[\mathrm{A} / \mathrm{B}] \equiv \log \left[\left(\mathrm{N}_{A} / \mathrm{N}_{B}\right)_{\text {star }} /\left(\mathrm{N}_{A} / \mathrm{N}_{B}\right)_{\text {Sun }}\right]$ 
since it is relatively easy to detect in the spectra of metal-poor stars and because it is predominantly produced in the $r$-process. It is seen in Figure 4 that near $[\mathrm{Fe} / \mathrm{H}]=-3$, (which includes some of the oldest stars in the Galaxy), the $[\mathrm{Eu} / \mathrm{Fe}]$ ratio reaches a peak of $\sim 50$ and varies from star-tostar by more than a factor of 100 . Thus, even though the $[\mathrm{Eu} / \mathrm{H}]$ ratios are still less than that of the sun, the relative ratio of this $r$-process element to iron in some of these stars is much larger than the solar ratio. It is also clear from the figure that this star-to-star scatter decreases with increasing $[\mathrm{Fe} / \mathrm{H}]$ tending toward younger (on average) stars. While there could be several possible explanations for this scatter, the most likely interpretation is that the early Galaxy was chemically inhomogeneous and unmixed.

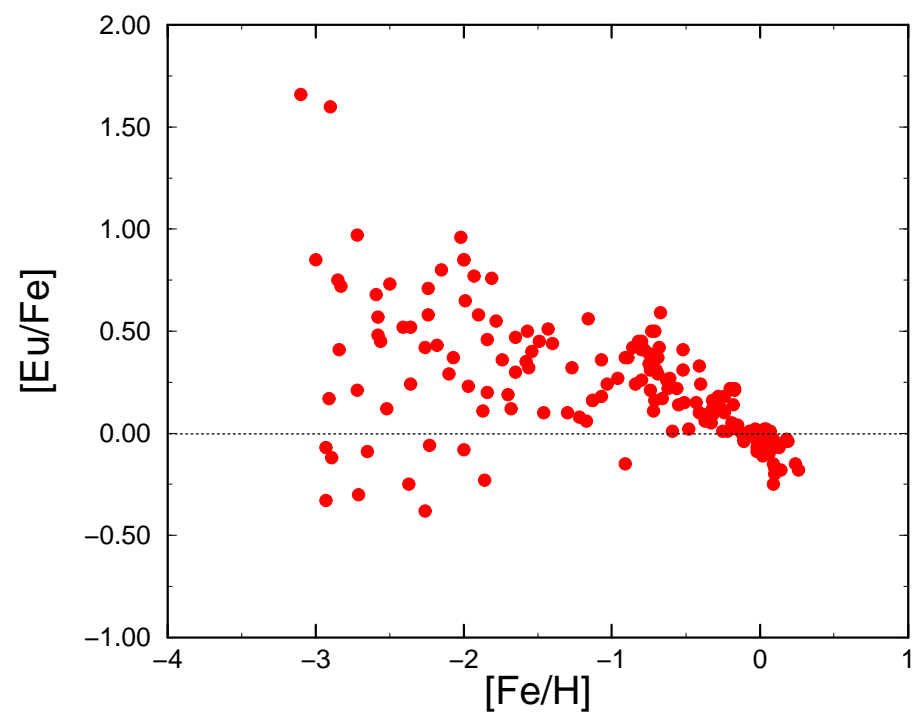

Figure 4. The ratio $[\mathrm{Eu} / \mathrm{Fe}]$ is plotted as a function of $[\mathrm{Fe} / \mathrm{H}]$ from various surveys of halo and disk stars $[1,2]$. The dotted line represents the solar value.

Abundance trends with metallicity also demonstrate the ongoing chemical evolution of the Galaxy. In the past $[\mathrm{Ba} / \mathrm{Eu}]$ has been employed to study the changing contributions to the $r$ - and $s$-processes. The element $\mathrm{Ba}$ is overwhelmingly synthesize in the $s$-process and provides a good indication of the $s$-process $n$-capture nucleosynthesis history of the Galaxy. There is, however, significant observationally related scatter in the Ba abundance data making it less reliable for such studies. As an alternative, new studies 
are now employing La, also a predominantly s-process element, to Eu to examine Galactic chemical evolution. Very preliminary results [22] seem to indicate some initial indication of the $s$-process below $[\mathrm{Fe} / \mathrm{H}]=-2$ with the major onset of the ejection of this material into the Galaxy occurring near a metallicity of -2 . These results lend support to earlier studies with similar findings $[10,23]$.

\section{Radioactive Chronometers and the Age of the Galaxy}

Abundance detections of certain long-lived radioactive isotopes can be employed as "chronometers" or clocks to determine the ages of the oldest stars. There have been a number of recent detections of the element thorium, with a half-life of $14 \mathrm{Gyr}$, in the metal-poor halo halo stars [4-8,24,25]. This element, along with uranium, is synthesized solely in the $r$-process. Comparison of the observed stellar abundance of this radioactive element with its initial (time-zero) abundance in an $r$-process site leads to a direct radioactive-age estimate of the star. We show in Figure 3 the abundance distribution, including Th, in the ultra-metal-poor star CS 22892052. While (as noted before) the heavy $n$-capture elements are consistent with the scaled solar $r$-process curve, the observed Th abundance lies below this same line. This difference is a clear demonstration that this star is older than the sun. To determine how much older requires knowledge of the initial Th abundance that must be predicted from $r$-process models. Such a model calculation is illustrated in Figure 3 by the dashed line [5]. The goal of such theoretical calculations is to reproduce the stellar, and hence the solar system $r$-process, abundance pattern and at the same time predict the abundances of the radioactive elements. Such predictions, to reduce errors, employ the ratio of Th to another $r$-process element, typically Eu. Utilizing this technique has led to chronometric age estimates ranging from $\simeq 11-15 \pm 4$ Gyr [4-8,24,25] that are consistent with globular cluster ages and cosmological age estimates based upon the observed supernova expansion rates. However, the $\mathrm{Th} / \mathrm{Eu}$ chronometer gives a very different and completely inconsistent age in the star CS 31082-001 [25]. This star was the first with a detection of $\mathrm{U}$ and the $\mathrm{Th} / \mathrm{U}$ chronometer does give an age of $15.5 \mathrm{Gyr}$ [26]. Since $\mathrm{Th} / \mathrm{Eu}$ and $\mathrm{Th} / \mathrm{U}$ give similar results in $\mathrm{BD}+17^{\circ} 3248$ [7], it is not clear yet why CS $31082-001$ is so different or if it is rare. Clearly additional $U$ detections will be needed to answer this question. Just as importantly reliable nuclear data, experimental where available but mostly theoretical predictions, for the neutron-rich nuclei in 
the $r$-process will be necessary to determine more accurately the ages of these old stars and put limits on the age of the Galaxy and the Universe.

\section{Acknowledgments}

Partial support for this research was provided by the National Science Foundation (AST-9986974 to JJC and AST-9987162 to CS) and by the Space Telescope Science grant GO-08342. JJC thanks the University of Texas at Austin Department of Astronomy John W. Cox Fund for partial support while this paper was being written.

\section{References}

1. C. Sneden and J. J. Cowan, Science, in press (2002).

2. J. W. Truran, J. J. Cowan, C. Sneden and C. Pilachowski, Publ. Astron. Soc. Pac., in press (2002).

3. N. Grevesse and A. J. Sauval, Space Sci. Rev. 85, 161 (1998).

4. C. Sneden, et al., Astrophys. J. 467, 819 (1996).

5. J. J. Cowan, et al., Ap. J. 521, 194 (1999).

6. C. Sneden, et al., Astrophys. J. 533, L139 (2000).

7. J. J. Cowan, et al., Astrophys. J. 572, 861 (2002).

8. J. Westin, C. Sneden, B. Gustafsson, and J. J. Cowan, Astrophys. J. 530, 783 (2000).

9. F. Käppeler, H. Beer, and K. Wisshak, Rep. Prog. Phys. 52, 945 (1989).

10. D. L. Burris, C. A. Pilachowski, T. A. Armandroff, C. Sneden, J. J. Cowan, and H. Roe, Astrophys. J. 544, 302 (2000).

11. C. Arlandini, et al., Astrophys. J. 525, 886 (1999).

12. G. J. Mathews, G. Bazan, and J. J. Cowan, Astrophys. J. 391, 719 (1992).

13. J. C. Wheeler, J. J. Cowan, and W. Hillebrandt, Astrophys. J. 493, L101 (1998).

14. Y. Ishimaru and S. Wanajo, Astrophys. J. 511, L33 (1999).

15. C. Sneden, et al., Astrophys. J. 566, L28 (2002).

16. D. Lambert and C. Allende-Prieto, MNRAS 335, 325 (2002).

17. G. J. Wasserburg, M. Busso, and R. Gallino, Astrophys. J. 466, L109 (1996).

18. C. Sneden, et al., in preparation (2002).

19. J. W. Truran and J. J. Cowan, in Nuclear Astrophysics, eds. W. Hillebrandt and E. Müller 64 (2000)

20. G. J. Wasserburg and Y.-Z. Qian, Astrophys. J. 529, L21 (2000).

21. A. G. W. Cameron, Astrophys. J. 562, 456 (2001).

22. J. Simmerer, et al., in preparation (2002).

23. K. K. Gilroy, C. Sneden, C. A. Pilachowski, and J. J. Cowan, Astrophys. J. 327, 298 (1988).

24. J. A. Johnson and M. Bolte, Astrophys. J. 554, 888 (2001).

25. R. Cayrel, et al., Nature 409, 691 (2001).

26. H. Schatz, et al., Astrophys. J. 579, 626 (2002). 